\title{
Mapping the antigenic diversification of SARS-CoV-2
}

Karlijn van der Straten ${ }^{1,2}$, Denise Guerra ${ }^{1}$, Marit J. van Gils ${ }^{1}$, Ilja Bontjer ${ }^{1}$, Tom G. Caniels ${ }^{1}$, Hugo D.G. van Willigen ${ }^{1}$, Elke Wynberg ${ }^{3}$, Meliawati Poniman ${ }^{1}$, Judith A. Burger ${ }^{1}$, Joey H. Bouhuijs $^{1}$, Jacqueline van Rijswijk ${ }^{1}$, Ayesha H.A. Lavell ${ }^{4}$, Brent Appelman ${ }^{5}$, Jonne J. Sikkens $^{4}$, Marije K. Bomers ${ }^{4}$, Alvin X. Han ${ }^{1}$, Brooke E. Nichols ${ }^{1,6}$, Maria Prins ${ }^{2,3}$, Harry Vennema $^{7}$, Chantal Reusken7, Menno D. de Jong ${ }^{1}$, Godelieve J. de Bree ${ }^{2}$, Colin A. Russell $^{1^{*}}$, Dirk Eggink ${ }^{1,7^{*}}$, Rogier W. Sanders ${ }^{1,8^{*}}$

${ }^{1}$ Department of Medical Microbiology \& Infection Prevention, Amsterdam UMC, University of Amsterdam, Amsterdam Institute for Infection and Immunity, Amsterdam, the Netherlands.

${ }^{2}$ Department of Internal Medicine, Amsterdam UMC, University of Amsterdam, Amsterdam Institute for Infection and Immunity, Amsterdam, the Netherlands.

${ }^{3}$ Department of Infectious Diseases, Public Health Service of Amsterdam, GGD, Amsterdam, the Netherlands.

4 Department of Internal Medicine, Amsterdam UMC, Vrije Universiteit Amsterdam, Amsterdam Institute for Infection and Immunity, Amsterdam, the Netherlands.

${ }^{5}$ Center for Experimental and Molecular Medicine, Amsterdam UMC, University of Amsterdam, Amsterdam Institute for Infection and Immunity, Amsterdam, the Netherlands. ${ }^{6}$ Department of Global Health, Boston University School of Public Health, Boston, USA

${ }^{7}$ Centre for Infectious Disease Control, National Institute for Public Health and the Environment, Bilthoven, the Netherlands.

${ }^{8}$ Department of Microbiology and Immunology, Weill Medical College of Cornell University, New York, USA.

* correspondence to:

c.a.russell@amsterdamumc.nl, dirk.eggink@rivm.nl, r.w.sanders@amsterdamumc.nl

\section{Abstract (max 150 words)}

Large-scale vaccination campaigns have prevented countless SARS-CoV-2 infections, hospitalizations and deaths. However, the emergence of variants that escape from immunity challenges the effectiveness of current vaccines. Given this continuing evolution, an important question is when and how to update SARS-CoV-2 vaccines to antigenically match circulating variants, similar to seasonal influenza viruses where antigenic drift necessitates periodic vaccine updates. Here, we studied SARS-CoV-2 antigenic drift by assessing neutralizing activity against variants-of-concern (VOCs) of a unique set of sera from patients infected with a range of VOCs. Infections with ancestral or Alpha strains induced the broadest immunity, while individuals infected with other VOCs had more strain-specific responses. Omicron was substantially resistant to neutralization by sera elicited by all other variants. Antigenic cartography revealed that all VOCs preceding Omicron belong to one antigenic cluster, while Omicron forms a new antigenic cluster associated with immune escape and likely requiring vaccine updates to ensure vaccine effectiveness. 
medRxiv preprint doi: https://doi.org/10.1101/2022.01.03.21268582; this version posted January 3, 2022. The copyright holder for this

preprint (which was not certified by peer review) is the author/funder, who has granted medRxiv a license to display the preprint in perpetuity.

All rights reserved. No reuse allowed without permission.

\section{Main text}

The COVID-19 pandemic, caused by the SARS-CoV-2 virus, represents an enormous threat to human health and burden to healthcare systems and economies worldwide. The unprecedented rapid development of efficacious vaccines fuelled hope of curtailing this pandemic and permitting a return to a society without societal restrictions. However, genetic drift of SARS-CoV-2 resulted in the emergence of multiple variants of concern (VOCs) with a higher transmissibility compared to the ancestral strain, and that challenge the effectiveness of public health measures, vaccines and/or therapeutics ${ }^{1}$. Based on this definition, the WHO designated the Alpha (Pango Lineage B.1.1.7), Beta (B.1.351), Gamma (P.1), Delta (B.1.617.2) and Omicron (B.1.1.529) variants as VOCs. The Alpha, Beta, Gamma and Delta VOCs have approximately 7 to 12 mutations in the Spike protein (S), while Omicron with 32 mutations has substantially more (Fig. 1A) $)^{1}$. Fifteen of Omicron's $S$ mutations are located in the Receptor Binding Domain (RBD), the most important antigenic site of S. Indeed, sera from COVID-19 patients infected with the ancestral strain and sera from vaccinees show up to 7fold and 4-fold reductions in neutralization activity against Beta and Gamma, while 20 to 40fold reductions are observed against Omicron ${ }^{2-5}$.

However, the precise antigenic relationships between these VOCs is unknown. Understanding the differences between the serological antibody responses elicited by these variants is important to assess the risk of re-infections after natural infection and breakthrough infections after vaccination. For seasonal influenza viruses, this type of antigenic data is combined with virus genetic and epidemiological data to quantify the evolution of the virus and guide annual updates of the seasonal influenza virus vaccines. Antigenic cartography can reliably quantify and visualize antigenic relationships between viral variants ${ }^{6,7}$ and is routinely used in influenza virus vaccines strain selection. So far, antigenic cartography for SARS-CoV2 has only been applied to cohorts of COVID-19 patients with uncertainty about their history of SARS-CoV-2 exposure and vaccination status ${ }^{8}$. In addition, the emergence of the Omicron variant clearly necessitates an update of these maps. Here, we studied the (cross-)neutralizing antibody responses in sera from a well-defined population of convalescent individuals with confirmed infections by the ancestral, Alpha, Beta, Gamma and Delta variants and used this data as input for antigenic cartography to map the antigenic evolution of SARS-CoV-2.

We collected and analysed a unique set of serum samples from 51 COVID-19 patients with a PCR-confirmed primary SARS-CoV-2 infection who did not receive COVID-19 vaccinations (Supplementary Table 1). Blood was drawn 3 to 9 weeks after symptom onset (median 40 days, range 24 to 63 days), which corresponds with the peak of the antibody response (Supplementary Table 1) ${ }^{9}$. Of the 51 participants, 31 were included with sequenceconfirmed VOC infection; $n=10$ for Alpha, $n=7$ for Beta, $n=4$ for Gamma and $n=10$ for Delta infected participants. 20/51 participants were assumed to be infected with the ancestral strain as they were sampled before the emergence of any VOC in the Netherlands.

We assessed the neutralizing capacity of the convalescent sera in a lentiviral-based pseudovirus neutralization assay against the ancestral strain, Alpha, Beta, Gamma, Delta and Omicron variants (Fig. 1a). The highest neutralization titres were generally measured against the homologous virus, as might be expected (Fig. 1a and Supplementary Fig. 1a). Only the Beta infected participants showed higher cross-neutralization titres against the Gamma variant compared to homologous neutralization. When comparing non-hospitalized patients, patients infected with the Alpha variant showed the strongest homologous neutralization (median of $1088 \mathrm{IU} / \mathrm{mL}$, range 73 to $2103 \mathrm{IU} / \mathrm{mL}$ ), followed by individuals infected with the Gamma strain (median of $156 \mathrm{IU} / \mathrm{mL}$, range 22 to $761 \mathrm{IU} / \mathrm{mL}$ ), the ancestral virus (median of $90 \mathrm{IU} / \mathrm{mL}$, range 28 to $237 \mathrm{IU} / \mathrm{mL}$ ) and the Delta variant (median of $85 \mathrm{IU} / \mathrm{mL}$, range 10 to $1635 \mathrm{IU} / \mathrm{mL}$ ) (Supplementary Fig. 1a). By contrast, none of the Beta infected participants showed strong homologous neutralization (median of $10 \mathrm{lU} / \mathrm{mL}$, range 10 to $24 \mathrm{lU} / \mathrm{mL}$ ).

Overall, the VOCs differed in their capacity to induce cross-neutralizing antibodies. Individuals infected with the Alpha variant induced the broadest response, followed by ancestral strain-infected, Gamma-infected and Delta-infected patients (Fig. 1b, Fig. 1c and Supplementary Fig. 1b), though there was substantial heterogeneity within all groups. None of the patients infected with the Beta variant showed substantial cross-neutralization activity. 
medRxiv preprint doi: https://doi.org/10.1101/2022.01.03.21268582; this version posted January 3, 2022. The copyright holder for this

preprint (which was not certified by peer review) is the author/funder, who has granted medRxiv a license to display the preprint in perpetuity.

All rights reserved. No reuse allowed without permission.

Reductions in neutralizing activity against Omicron were substantial in all groups. Omicron neutralization dropped below the limit of detection $\left(2 \mathrm{IU} / \mathrm{mL}\right.$ or an $\mathrm{ID}_{50}$ of 20 ) in 21/51 of the studied individuals. The fold-reduction of Omicron neutralization versus homologous neutralization was 9-fold (range 0.5 to 93-fold) when considering all patients, 10-fold (range 3 to 93-fold) for patients infected with ancestral strains, 56-fold (range 0.5 to 89-fold) for Alpha, 6-fold (range 1 to 22-fold) for Gamma, and 5-fold (range 1 to 51-fold) for Delta infected patients.

To explore the antigenic relationships between the VOCs, we used the neutralization data to construct a SARS-CoV-2 antigenic map (Fig. 2a). In this map, homologous sera tend to cluster around the infecting strain, reflecting that homologous neutralization is dominant. The ancestral and Alpha viruses cluster tightly together in the centre of the map, while the Beta, Gamma, and Delta variants all lie within 2 antigenic units ( 1 unit $=2$-fold change in neutralization titre) of the ancestral virus suggesting a high degree of antigenic similarity. For influenza viruses, variants are considered to be antigenically similar in case of antigenic distances below 3 antigenic units, i.e. an 8-fold change in neutralization titre, and different when above this threshold ${ }^{10,11}$. By analogy the ancestral, Alpha, Beta, Gamma and Delta variants belong to one antigenic cluster. However, the distance between this antigenic cluster and Omicron is more than 5 antigenic units (>32-fold change in neutralization) implying that Omicron represents a first widely circulating new major SARS-CoV-2 variant (Fig. 2a). One caveat is that it is unclear whether 2-fold changes in pseudovirus neutralization titres are directly comparable to 2-fold changes in hemagglutination inhibition assay titres used to characterize influenza viruses. However, the change in neutralization between Omicron and other variants of SARS-CoV-2, including the ancestral strain, is striking.

We next used neutralization data from sera of 122 COVID-19 naïve vaccinees receiving either two Moderna (mRNA-1273, $n=30$ ), Pfizer/BioNTech (BNT162b2, $n=49$ ), or AstraZeneca (AZD1222, $n=30$ ) vaccines, which are all based on the ancestral S sequence to generate a second antigenic map ${ }^{4}$. This map agreed well with the infectee map (Fig. 2b), and corroborated that Omicron represents a distinct antigenic variant from viruses currently included in vaccines. Interestingly, while the distributions of sera from recipients of different vaccines overlap, there is a skew of sera of mRNA-1273 vaccinees towards Omicron suggesting small differences in antigen stimulation among vaccine formulations considered here.

We have started to define the antigenic SARS-CoV-2 landscape after two years of antigenic drift, which should inform risk assessment of re-infections as well as strain selection for COVID-19 vaccine updates. We can draw several conclusions. First, homologous neutralization was usually stronger than heterologous neutralization. Second, heterologous responses were broadest and most potent in individuals infected with Alpha and ancestral strains, while infection with Delta resulted in narrow-specificity responses. Third, crossneutralization of Omicron was weak across all samples, with few exceptions. Moreover, the ancestral and Alpha strains are at the centre of our antigenic map, which supports the use of the current COVID-19 vaccines based on the ancestral strain, in case of circulation of the Alpha, Beta, Gamma and Delta variants. Our data suggest that updated vaccines based on the Beta or Delta variants would not have been appreciably more effective than the ancestral virus-based vaccines. However, the substantial reduction of neutralization in all groups against the Omicron variant indicates a high risk of re-infections and vaccine breakthrough cases when exposed to this VOC. The long antigenic distance between Omicron and the preceding variants in the antigenic map indicates that the current high rates of Omicron infections are at least partially associated with immune escape and that a vaccine update is required.

As in the case of seasonal influenza viruses, the prospect of SARS-CoV-2 becoming an endemic virus with recurring outbreaks implies the need for surveillance of antigenic drift and yearly administration of updated vaccines, especially for individuals at risk for severe COVID-19. Antigenic cartography efforts such as those presented here, can inform future vaccine updates. 
medRxiv preprint doi: https://doi.org/10.1101/2022.01.03.21268582; this version posted January 3, 2022. The copyright holder for this

preprint (which was not certified by peer review) is the author/funder, who has granted medRxiv a license to display the preprint in perpetuity.

All rights reserved. No reuse allowed without permission.

\section{Acknowledgements}

We thank all public health services (GGD) in the Netherlands for their help in contacting participants. We are also thankful to the study personnel and the participants of the COSCA, RECoVERED and the S3-study for their contribution to this research.

Conflicts of interest: C.A.R. reports paid advisory roles for Sanofi Pasteur on the epidemiology of influenza viruses and SARS-CoV-2 and for Moderna on the evolution of SARS-CoV-2. None of the other authors have conflicts of interest related to this research.

Funding. R.W.S. and C.A.R. are recipients of Vici grants from the Netherlands Organization for Scientific Research (NWO). C.A.R. and A.X.H. are also supported by an ERC Consolidator Award. This work was supported by the NOW ZonMw grant agreement no. 10150062010002 to M.D.d.J., and 10430072110003 to G.J. de Bree and the Public Health Service of Amsterdam Research \& Development grant number 21-14 to M. Prins (RECoVERED). J.J.S. and M.K.B. are recipients of the NOW grant agreement no. 10430022010023 to J.J.S. and M.K.B.

\section{Figure legends}

Figure 1. SARS-CoV-2 genetic diversity and neutralization. a. Molecular models of SARSCoV-2 S protein highlighting the locations of mutations in the ancestral strain (D614G, blue), Alpha (green), Beta (yellow), Gamma (orange), Delta (red) and Omicron (magenta) VOCs. b. Midpoint neutralization titres against the VOCs in International Units per $\mathrm{mL}(\mathrm{IU} / \mathrm{mL})$. The individuals are grouped per VOC and plotted accordingly. The grey bar $(10 \mathrm{lU} / \mathrm{ml})$ indicates the neutralization cut-off for all strains except Omicron (cut-off $2 \mathrm{IU} / \mathrm{mL}$ ). Non-hospitalized patients are indicated with dots and hospitalized patients with triangles. The homologous neutralization is highlighted using a bar. The Wilcoxon signed rank test with Benjamini Hochberg correction was used to compare cross-neutralization titres with the homologous neutralization. ns $=$ non-significant, ${ }^{*}=p<0.005,{ }^{* *}=p<0.01,{ }^{* * * *}=p<0.0001$. c. Spider plot of the median neutralization titre $(\mathrm{IU} / \mathrm{mL})$ of each group against all VOCs. A cut-off of $10 \mathrm{IU} / \mathrm{mL}$ is used for all strains. Colouring corresponds with figure $1 \mathrm{a}$ and $\mathrm{b}$.

Figure 2. SARS-CoV-2 antigenic cartography. a. Antigenic map of SARS-CoV-2 VOCs based on post-SARS-CoV-2 infection sera. SARS-CoV-2 VOCs are shown as circles and sera are indicated as squares. Each square corresponds to sera of one individual and is coloured by the infecting SARS-CoV-2 variant. Both axes of the map are antigenic distance and each grid square (1 antigenic unit) represents a two-fold change in neutralization titre. The distance between points in the map can be interpreted as a measure of antigenic similarity of similarity in reactivity where closer together points are more similar. b. Antigenic map of SARS-CoV-2 VOCs based on post-vaccination sera from individuals without prior SARS-CoV-2 infections. Each serum is coloured by the vaccine that individual received.

Supplementary Figure 1. Homologous and cross-neutralization. a. Midpoint neutralization titres against the VOCs in International Units per $\mathrm{mL}(\mathrm{IU} / \mathrm{mL})$. The individuals are grouped per VOC they were infected with and plotted accordingly. Non-hospitalized patients (dots) showed lower neutralization response compared to hospitalized patients (triangles) as in line with previous research ${ }^{12}$. A Mann-Whitney test is used to test for differences between group medians (line). ns $=$ non-significant, ${ }^{*}=p<0.005,{ }^{* * *}=p<0.0001$. b. Cross-neutralization is expressed as the geometric mean of the neutralization titres against all VOCs except the autologous strain in $\mathrm{IU} / \mathrm{mL}$. A cut-off of $10 \mathrm{IU} / \mathrm{mL}$ is used for all neutralization titres, as indicated by the grey bar. 
medRxiv preprint doi: https://doi.org/10.1101/2022.01.03.21268582; this version posted January 3, 2022. The copyright holder for this preprint (which was not certified by peer review) is the author/funder, who has granted medRxiv a license to display the preprint in perpetuity.

All rights reserved. No reuse allowed without permission.

\section{References:}

1. World Health Organization. Tracking SARS-CoV-2 variants (2021).

2. Caniels, T.G., et al. Emerging SARS-CoV-2 variants of concern evade humoral immune responses from infection and vaccination. Sci Adv 7, eabj5365 (2021).

3. Garcia-Beltran, W.F., et al. mRNA-based COVID-19 vaccine boosters induce neutralizing immunity against SARS-CoV-2 Omicron variant. medRxiv, 2021.2012.2014.21267755 (2021).

4. van Gils, M.J., et al. Four SARS-CoV-2 vaccines induce quantitatively different antibody responses against SARS-CoV-2 variants. medRxiv, 2021.2009.2027.21264163 (2021).

5. Wilhelm, A., et al. Reduced Neutralization of SARS-CoV-2 Omicron Variant by Vaccine Sera and monoclonal antibodies. medRxiv, 2021.2012.2007.21267432 (2021).

6. Smith, D.J., et al. Mapping the Antigenic and Genetic Evolution of Influenza Virus. Science 305, 371-376 (2004).

7. Fonville, J.M., et al. Antibody landscapes after influenza virus infection or vaccination. Science 346, 996-1000 (2014).

8. Liu, C., et al. Reduced neutralization of SARS-CoV-2 B.1.617 by vaccine and convalescent serum. Cell 184, 4220-4236.e4213 (2021).

9. Long, Q.-X., et al. Antibody responses to SARS-CoV-2 in patients with COVID-19. Nature Medicine 26, 845-848 (2020).

10. Barr, I.G., et al. WHO recommendations for the viruses used in the 2013-2014 Northern Hemisphere influenza vaccine: Epidemiology, antigenic and genetic characteristics of influenza $A(\mathrm{H} 1 \mathrm{~N} 1)$ pdm09, $\mathrm{A}(\mathrm{H} 3 \mathrm{~N} 2)$ and $\mathrm{B}$ influenza viruses collected from October 2012 to January 2013. Vaccine 32, 4713-4725 (2014).

11. Prevention, C.o.D.C.a. Antigenic Characterization. (2021).

12. Lynch, K.L., et al. Magnitude and Kinetics of Anti-Severe Acute Respiratory Syndrome Coronavirus 2 Antibody Responses and Their Relationship to Disease Severity. Clin Infect Dis 72, 301-308 (2021).

13. Grobben, M., et al. Cross-reactive antibodies after SARS-CoV-2 infection and vaccination. medRxiv, 2021.2005.2026.21256092 (2021).

14. Wynberg, E., et al. Evolution of COVID-19 symptoms during the first 12 months after illness onset. Clin Infect Dis (2021).

15. Schmidt, F., et al. Measuring SARS-CoV-2 neutralizing antibody activity using pseudotyped and chimeric virusesSARS-CoV-2 neutralizing antibody activity. Journal of Experimental Medicine 217(2020).

16. Kristiansen, P.A., et al. WHO International Standard for anti-SARS-CoV-2 immunoglobulin. The Lancet 397, 1347-1348 (2021). 
medRxiv preprint doi: https://doi.org/10.1101/2022.01.03.21268582; this version posted January 3, 2022. The copyright holder for this preprint (which was not certified by peer review) is the author/funder, who has granted medRxiv a license to display the preprint in All rights reserved. No reuse allowed without permission.

\section{Method section}

\section{Study population}

51 adults with a PCR proven primary SARS-CoV-2 infection were included in the COSCAstudy or the RECOVERED study between June 2020 and July 2021 at Amsterdam UMC and via the Dutch national SARS-CoV-2 sequence surveillance program as described previously ${ }^{13,14}$. 3-9 weeks after symptom onset, blood, patient demographics, time between symptom onset and sampling, and admission status were collected. The diagnostic oropharyngeal swabs were used to determine the SARS-CoV-2 strain causing the infection. All ancestral strain infected participants $(n=20)$ were assumed to be infected with the ancestral strain as they were sampled before the emergence of any VOC in the Netherlands. Two of the individuals infected with Alpha strains harboured the E484K mutation, which are indicated as squares in all graphs. The Omicron variant did not yet circulate at time of inclusion.

Neutralization data on COVID-19 naive vaccinee sera collected in the S3-study, were kindly provided by van Gils et al ${ }^{4}$. Post-vaccination sera was obtained four weeks after the second doses of either Moderna (mRNA-1273), Pfizer/BioNTech (BNT162b2), or AstraZeneca (AZD1222). Post-vaccination serum after Janssen (Ad26.COV2.S) were excluded from analysis because they did not have enough non-threshold titres to be included in the map.

The COSCA study, the RECoVERED study and the S3-study were conducted at the Amsterdam University Medical Centres, the Netherlands, and approved the medical ethical review board of the Amsterdam University Medical Centres (NL 73281.018.20, NL73759.018.20, NL73478.029.20, respectively). All individuals provided written informed consent before participating.

\section{Pseudovirus design}

The Alpha, Beta, Gamma, Delta and Omicron pseudovirus constructs contained the following mutations: deletion $(\Delta)$ of H69, V70 and Y144, N501Y, A570D, D614G, P681H, T716I, S982A, and D1118H in Alpha; L18F, D80A, D215G, L242H, R246I, K417N, E484K, N501Y, D614G, and A701V in Beta; and L18F, T20N, P26S, D138Y, R190S, K417T, E484K, N501Y, D614G, H655Y, and T1027I in Gamma; T19R, G142D, E156G, 1 157-158, L452R, T478K, D416G, P681R and D950N in Delta; and A67V, $\Delta 699-70$, T95I, G142D, $\Delta 143-145, \Delta 211$, L212I, ins214EPE, G339D, S371L, S373P, S375F, K417N, N440K, G446S, S477N, T478K, E484A, Q493K, G496S, Q498R, N501Y, Y505H, T547K, D614G, H655Y, N679K, P681H, N764K, D796Y, N856K, Q954H, N969K, L981F in Omicron. The ancestral strain included the D614G mutation as this variant emerged early in the pandemic and all ancestral infected participants are most likely to be infected with this variant. The spike constructs were ordered as gBlock gene fragments (Integrated DNA Technologies) and cloned Sacl and Apal in the pCR3 SARSCoV-2-S $S_{\triangle 19}$ expression plasmid (GenBank: MT449663.1) using Gibson Assembly (Thermo Fisher Scientific). The pseudovirus constructs were made using the QuikChange Site-Directed Mutagenesis Kit (Agilent Technologies) and verified by using Sanger sequencing. Pseudoviruses were procedures by cotransfecting HEK293T cells (American Type Culture Collection, CRL-11268) with the pCR3 SARS-CoV-2-S ${ }_{\triangle 19}$ expression plasmid and the pHIV$1_{\mathrm{NL} 43} \Delta$ Env-NanoLuc reporter virus plasmid. 48 hours after the transfection, cell supernatants containing the pseudovirus were harvested and stored at $-80^{\circ} \mathrm{C}$ until further use. 
medRxiv preprint doi: https://doi.org/10.1101/2022.01.03.21268582; this version posted January 3, 2022. The copyright holder for this preprint (which was not certified by peer review) is the author/funder, who has granted medRxiv a license to display the preprint in perpetuity.

All rights reserved. No reuse allowed without permission.

\section{SARS-CoV-2 pseudovirus neutralization assay}

The pseudovirus neutralization assay was performed as described previously ${ }^{2}$. Shortly, HEK293T/ACE2 cells were kindly provided by $P$. Bieniasz ${ }^{15}$ were seeded at a density of 20,000 cells per well in a 96-well plate coated with poly-lysine $(50 \mathrm{~g} / \mathrm{ml}) 1$ day before the start of the neutralization assay. The next day, heat-inactivated sera samples were serial diluted in threefold steps, starting at 1:20 dilution to test for Omicron pseudovirus neutralization and $1: 100$ for the other variants and the ancestral pseudovirus. Sera was diluted in cell culture medium (DMEM), supplemented with $10 \%$ fetal bovine serum, penicillin $(100 \mathrm{U} / \mathrm{ml})$, streptomycin $(100 \mathrm{~g} / \mathrm{ml})$, and GlutaMAX (Gibco), mixed in a 1:1 ratio with pseudovirus, and incubated for 1 hour at $37^{\circ} \mathrm{C}$. These mixtures were then added to the cells in a 1:1 ratio and incubated for 48 hours at $37^{\circ} \mathrm{C}$, followed by a PBS wash, and lysis buffer was added. The luciferase activity in cell lysates was measured using the Nano-Glo Luciferase Assay System (Promega) and GloMax system (Turner BioSystems). Relative luminescence units were normalized to those from cells infected with SARS-CoCV-2 pseudovirus in the absence of sera. The inhibitory neutralization titres (ID50) were determined as the serum dilution at which infectivity was inhibited by $50 \%$, using a nonlinear regression curve fit (GraphPad Prism software version 8.3). The International Standard for anti-SARS-CoV-2 immunoglobulins provided by the $\mathrm{WHO}^{16}$ were used to convert the ID50 values into International Units per milliliters $(\mathrm{IU} / \mathrm{mL})$. Samples with $\mathrm{IU} / \mathrm{mL}$ titres $<10$ were defined as having undetectable neutralization against the ancestral, Alpha, Beta, Gamma and Delta variant. For Omicron neutralization, the start-dilution of 1:20 enables a cut-off of $<2 \mathrm{IU} / \mathrm{mL}$.

\section{Antigenic cartography}

Antigenic maps were constructed as previously described ${ }^{6,7}$ using the antigenic cartography software from https://acmacs-web.antigenic-cartography.org. In brief, this approach to antigenic mapping uses multidimensional scaling to position antigens (viruses) and sera in a map to represent their antigenic relationships. The maps there relied on the first SARS-CoV2 infection serology data and post-vaccination serology data for the maps shown in Figure $2 a$ and Figure $2 \mathrm{~b}$. The positions of antigens and sera were optimized in the map to minimise the error between the target distances set by the observed pairwise virus-serum combinations in the pseudovirus assay described above and the resulting computationally derived map. Maps were constructed in 2, 3, 4, and 5 dimensions to investigate the dimensionality of the antigenic relationships. Both the infectee and vaccinee datasets were strongly two-dimensional with only small improvements in residual mean squared error of the maps as map dimensionality increased.

\section{Visualization and statistical analysis}

Data visualization and statistical analyses were performed in GraphPad Prism software (version 8.3). Mann-Whitney test was used for non-paired group comparisons. Wilcoxon signed rank test with Benjamini Hochberg correction was used to compare cross-neutralization titres with the homologous neutralization. Spider plots were made in Excel 2016. Fold-changes were calculated using a cut-off of $10 \mathrm{lU} / \mathrm{mL}$ for all VOCs. The antigenic maps were produced using the antigenic cartography software mentioned above. 
Figure 1. SARS-CoV-2 genetic diversity and neutralization

a

Ancestral strain

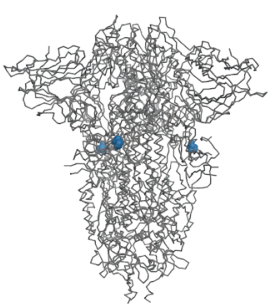

0

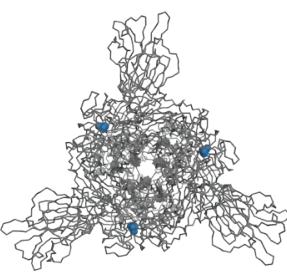

Alpha (B.1.1.7)

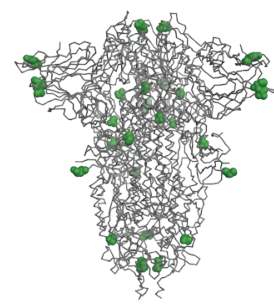

0
Beta (B.1.351)

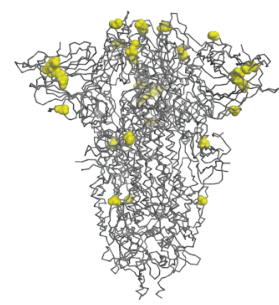

8

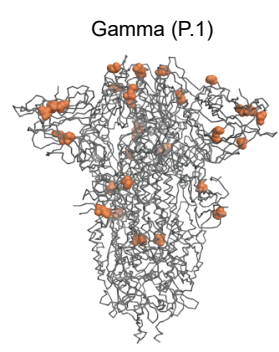

$\theta$

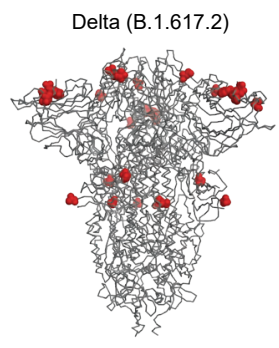

$\theta$

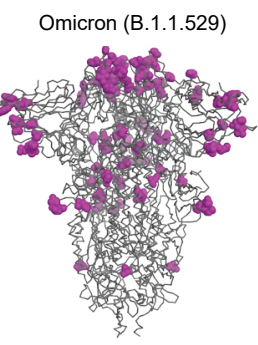

$\theta$
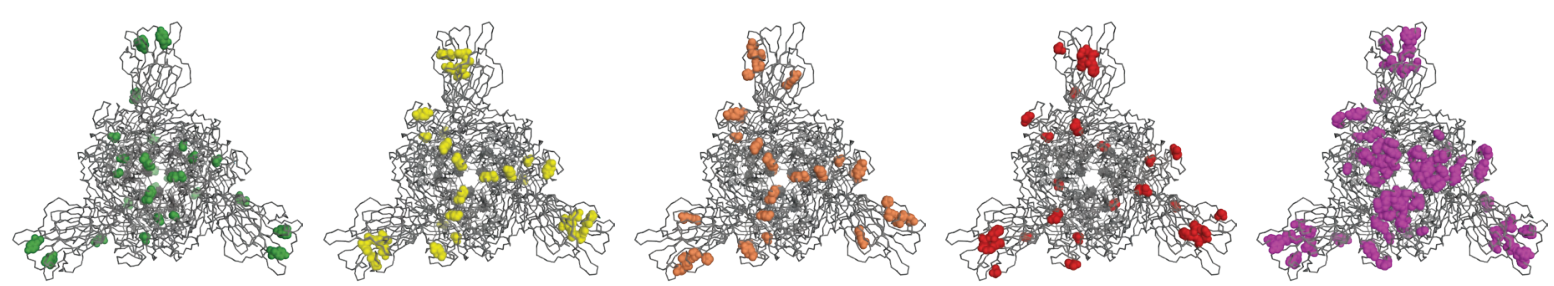

b

Ancestral strain-infected
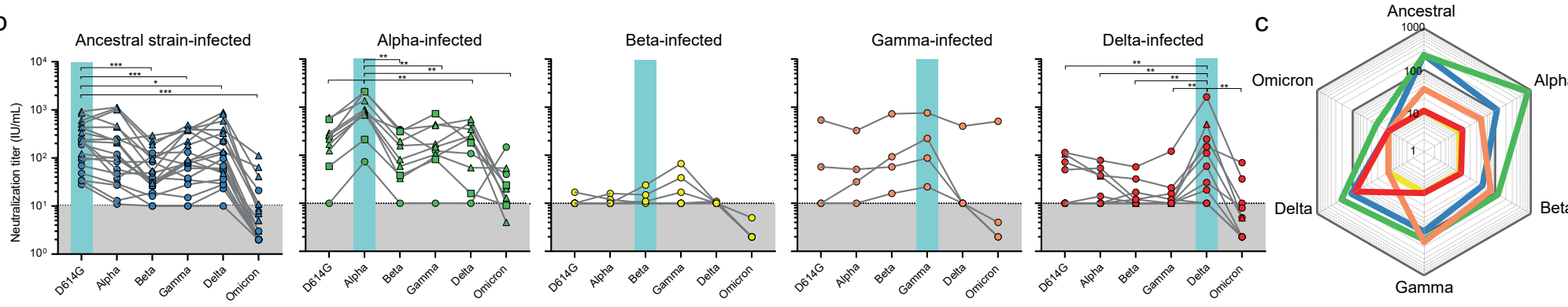
Figure 2. SARS-CoV-2 antigenic cartography

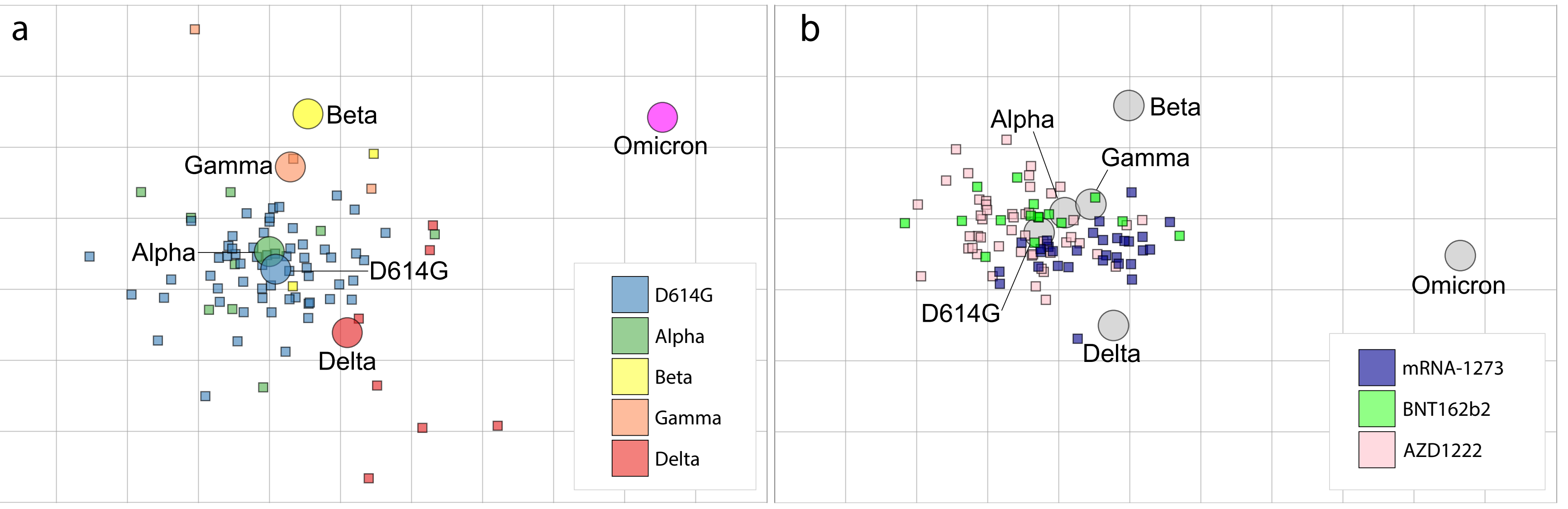

